\title{
Gödel, Tarski, Turing and the conundrum of free will
}

\author{
Chetan S. Mandayam Nayakar and R. Srikanth \\ Poornaprajna Institute of Scientific Research, Sadashivnagar, Bangalore and \\ Raman Research Institute, Sadashivnagar, Bangalore
}

\begin{abstract}
The problem of defining and locating free will $(\mathrm{FW})$ in physics is studied. On basis of logical paradoxes, we argue that FW has a meta-theoretic character, like the concept of truth in Tarski's undefinability theorem. Free will exists relative to a base theory if there is freedom to deviate from the deterministic or indeterministic dynamics in the theory, with the deviations caused by parameters (representing will) in the meta-theory. By contrast, determinism and indeterminism do not require meta-theoretic considerations in their formalization, making FW a fundamentally new causal primitive. FW exists relative to the meta-theory if there is freedom for deviation, due to higher-order causes. Absolute free will, which corresponds to our intuitive introspective notion of free will, exists if this meta-theoretic hierarchy is infinite. We argue that this hierarchy corresponds to higher levels of uncomputability. In other words, at any finitely high order in the hierarchy, there are uncomputable deviations from the law at that order. Applied to the human condition, the hierarchy corresponds to deeper levels of the subconscious or unconscious mind. Possible ramifications of our model for physics, neuroscience and artificial intelligence (AI) are briefly considered.
\end{abstract}

\section{INTRODUCTION}

Informally speaking, $\mathrm{FW}$ is the power to choose one alternative from many. We think that we have it. Yet its existence and nature have been debated for over two thousand years by philosophers, scientists and theologians [1, 2]. Free will is, arguably, a familiar stranger. One basic difficulty here is its paradoxical nature: FW incorporates two opposing notions: freedom and control. On the one hand, freedom suggests indeterminism; while control suggests intent and determinism. From this perspective, FW is an oxymoron. There are some easy questions to ask about it that are hard to answer:

1. What is $F W$ ? Physicists typically treat it as unpredictability or uncorrelatedness with some past data. By this criterion, uncontrolled or unreasonable behavior would qualify as free, and thus does not work. On the other hand, if control and predictability are the requirements, then the altruism of a saint and the selfish actions of a materialist are equally predictable, though intuitively, the former is the one we would deem free. The relevant question here is: how to define FW?

2. Does it exist is the world? Introspection suggests that we are free in the sense that we could have done other than what we did. But unless FW is well defined, this intuitive feeling may well be illusory!

3. Is it compatible with the laws of physics? The answer to this would depend on those to the above two questions!

4. What is its neurological basis, if FW exists? Here again, the answer depends on the answer to the above three.

This article addresses the first three questions above. The last is dealt with by us elsewhere [3]. There are three broad metaphysical positions on FW: Compatibilism, which holds that determinism is compatible with FW. A person may choose freely and yet an omniscient being may possess foreknowledge of that choice. The opposite view is incompatibilism, according to which FW and determinism are incompatible. Two divergent incompatibilist views are hard determinism, which rejects FW in favor of determinism, and libertarianism, which rejects determinism in favor of FW. To the determinist, free will is at best illusory. The variety of FW that arguably poses the greatest challenge is the liberatarian one, and it is this that we consider in this work.

The remaining article is structured as follows. In Section II we briefly review the current status of free will in quantum mechanics. In Section ஹII we present the Weak Free Will paradox, which is concerned with question of the compatibility of FW with the laws of physics. A recapitulation of a model of FW we developed in Ref. [4] in response to the Weak Free Will paradox is presented in Section IV The Strong Free Will paradox, the argument that the only causal primitives in Nature are indeterminism and indeterminism, is presented in Section $\nabla$. In response to the latter paradox, FW is proposed in Section VI as an infinite causal hierarchy of meta-laws, where higher-order laws can cause deviations in lower-order ones. The orders of the hierarchy are then differentiated by different levels of uncomputability in Section VII] A justification for this association is presented in Section VIII] The fuller implications for neuroscience, physics and AI are discussed in Section IX. Finally, we conclude in Section X. 


\section{QUANTUM MECHANICS AND FW}

Quantum mechanics, by introducing the new paradigm of intrinsic randomness provided fresh impetus to the FW debate. At first it seems that quantum indeterminism provides room to accomodate free will. Yet, randomness also means loss of control [5]. Two kinds of freedom are sometimes discerned: that of experimenters who choose measurement settings, and of particles that decide the measurement outcome [6]. T' Hooft [7] proposes identifying FW with freedom to modify the initial state of a system.

Reconsideration of randomness in light of quantum nonlocality has provided new insights. Gisin [8] has argued that the Many-Worlds interpretation [9] of quantum mechanics is incompatible with quantum nonlocality and the existence of FW. The Conway-Kochen FW Theorem [10, 11] proves that given the violation of Bell-type inequality and relativistic time-ordering, the assumption of freedom of choice of settings by observers implies a similar 'free will' on the part of particles, i.e., independence from all past information (with 'past' defined as the past light cone or all complement of the future light cone). Particle outcomes are then acts of creation or becoming rather than pre-determinate values [12]. The claim [10, 11] that the freedom of experimenters and the violation of Belltype inequalities rule out covariant stochastic dynamical explanations of quantum nonlocality was disputed by others [13, 14]. A reconciliation of these two positions can be brought about by the observation [12, 15] that whereas 'collapse' of the nonlocal wavefunction leading to correlated outcomes is not covariant, still the 'cloud of future events' is. Hall [16] has refined the notion of FW discussed in the Free Will theorem, by obtaining a trade-off between the freedom of observers or measurement independence and the freedom of particles or indeterminism in measurement outcomes.

Suarez [17] has argued that assuming freedom of the experimenter in a measurement configuration where two observers perform a Bell inequality test [18], such that each considers her/his own measurement as having been performed first, then time-ordering of physical causation must be given up. Outcomes are then controlled by an immaterial agency outside space-time that determines the order of outcomes without affecting the probabilities, given by the Born rule. By this argument, FW and randomness can coexist [19].

\section{THE WEAK FW PARADOX}

Consider world $W$ governed by deterministic physical law, $L$. All objects in $W$ evolve according to $L$. The pair $(W, L)$ constitute theory $\mathcal{T}$. By determinism, the physical state $\psi_{t}$ at time $t$ of an inanimate agent (e.g., a particle) and the physical law determine the state $\psi_{t+1}$ at subsequent time $t+1: \psi_{t+1}=L\left(\psi_{t}\right)$. Free will seems to entail for a conscious agent $X$ in $W$, that in general,

$$
\psi_{t+1} \neq L\left(\psi_{t}\right)
$$

meaning that $X$ 's behavior is not captured by $L$. We may consider replacing $L$ by a probabilistic law $L^{\prime}$ such that

$$
\Psi_{t+1}=L^{\prime}\left(\Psi_{t}\right)
$$

where $\Psi_{t+1}$ is a random variable representing probabilitistic evolution, characterized by mean $\mu$ and standard deviation $\sigma$. By the Law of Large Numbers, the sample mean $\left\langle\Psi_{t+1}\right\rangle^{(n)}$ of the random variable $\Psi_{t+1}$, over $n$ trials satisfies

$$
\lim _{n \rightarrow \infty} \operatorname{Pr}\left(\left|\left\langle\Psi_{t+1}\right\rangle^{(n)}-\mu\right|>\epsilon\right)=0
$$

In other words, it is exponentially unlikely that the agent $X$ will, over many trials, choose atypical sequences. There is thus long-run determinism and on that scale, lack of freedom.

Thus libertarian FW is compatible with neither a deterministic nor indeterministic physical law. This is the Weak Free Will paradox. We conclude that, libertarian FW, if it exists for an agent $X$, must be a supra-physical commodity that causes deviations from the laws of the world physical $W$ in which $X$ (physically) lives. Causality is no longer closed under physics in a world where FW exists. Otherwise- if free-willed action were explainable by $L-$, then there is no deviation by definition, and FW must be illusory.

The quantum homeostatis hypothesis [17] is another response to the Weak Free Will paradox. According to it, a conscious agent may exert FW by controlling the order of outcomes, without contradicting the probability law Eq. (2). In order not to deviate from the long term distribution required by $L^{\prime}$, periods of deliberate, conscious action must be compensated by other periods of uncontrolled behavior (e.g., sleep).

\section{FREE WILL AS THE POWER OF MIND OVER MATTER}

A proposal that incorporates will-driven deviations from the physical law, as required in our resolution of the Weak Free Will paradox, is the Freedom-Understanding-Nature (FUN) model [4]. An agent is assumed to be equipped 
with two possibly opposing faculties: Nature $(N)$, determined by physical law $L$ via brain (limbic) dynamics, genetic proclivities and instinctual drives, and Understanding $(U)$, determined by higher cortical faculties of morality and compassion. Here $N$ is a manifestation of $L$ from which will-driven deviations may occur. For example, $N$ may manifest as a psycho-somatic desire that urges the agent to procure a physical object, while $U$ may advise restraint. Freedom $(F)$ is an immaterial resource that empowers $X$ to deviate choice $C$ away from the option urged on by $N$ towards the one advised by $U$. This deviation cannot be captured by a Hamiltonian dynamics or a mechanical model, since these exist within $L$. The base-law of the deviation corresponds to the known physical law (here $N$ ), but the parameters of deviation (here $\varphi$ and possibly $U$ ) are associated with the meta-law. Together the meta-law (which encompasses $L$ ) and parameters associated with it consistute the meta-theory.

Free will is the power to conform to $U$, deviating from $N$ if necessary. A simple mathamatical model: suppose agent $X$ is faced with a two-valued choice "0" or " 1 ". $U$ and $N$ are represented by random variables $\hat{U}$ and $\hat{N}$ that take values in dichotomic choice space of $\Omega \equiv\{0,1\}$. Let $U$ advise option " 0 " whereas $N$ urges $X$ towards option "1" with a 'strength' $\nu$. The eventual choice $C$ of the agent is represented by random variable $\hat{C}$, which is a convex combination of $\hat{U}$ and $\hat{N}$, with the weight assigned to $U$ determined by the freedom parameter $\varphi$ (where $0 \leq \varphi \leq 1$ ), representing $F$. Thus:

$$
\hat{C} \equiv(1-\varphi)\left(\begin{array}{c}
1-\nu \\
\nu
\end{array}\right)+\varphi\left(\begin{array}{l}
1 \\
0
\end{array}\right)=\left(\begin{array}{c}
\varphi+(1-\varphi)(1-\nu) \\
(1-\varphi) \nu
\end{array}\right)
$$

In Eq. (4), the larger is $\varphi$, the more can $C$ deviate from $N$ towards $U$. If freedom is maximal, i.e., $\varphi=1$, then the agent behaves deterministically (mathematically, $\hat{C}$ is pure), demonstrating that $\mathrm{FW}$ is distinct from unpredictability in this model. We note that $\hat{C}$ becomes more random as $\varphi$ drops from 1 .

Free will $\Phi$ is quantified as a measure of closeness of $\hat{U}$ to $\hat{C}$ normalized by the distance of $\hat{U}$ from $\hat{N}$.

$$
\Phi=(\vec{C} \cdot \vec{U})|\vec{U}-\vec{N}|
$$

where $\vec{C}(\vec{N})$ is a vector representation of $\hat{C}(\hat{N})$ and $|\cdot|$ denotes trace distance. By definition, $0 \leq F \leq 1$. For Eq. (4), we find

$$
\Phi=\frac{\varphi}{2}(\varphi+(1-\varphi)(1-\nu))|1-2 \varphi| .
$$

We may thus think of FW $\Phi$ as innate freedom $F$ (represented by $\varphi$ ) expressed physically when there is a conflict between $U$ and $N$. The greater the conflict, the greater the potential for this expression. Here innateness means that $F$ is a part of the meta-theory (or higher-order theory), while its expression is at the base, physical level.

\section{THE STRONG FW PARADOX}

That libertarian FW produces deviations from the base law, only tells how FW acts. It does not clarify what this commodity is. We now attempt to define FW as the resource that can produce such deviations. The Strong Free Will paradox, given here, is concerned with the question of whether these deviations themselves are lawful according to a higher law. Suppose agent $X$ is faced with a situation that presents a choice. By our resolution of the Weak Free Will paradox, in free choice, physical causation fails in $W$ in that $X$ may deviate from the physical law $L . X$ has FW relative to $W$. The parameters of this deviation are not governed by $L$, and therefore are objects that live in the extended world $W^{+}$. One now considers whether these parameters themselves are lawful or free-willed. For agent $X$ to have genuine FW, these deviation parameters should have been freely chosen.

One possibility is that there there are deterministic laws $L^{+}$in $W^{+}$that explain the causes that produce the deviation on the base law $L$. We then have:

$$
\psi_{t+1}^{+}=L^{+}\left(\psi_{t}^{+}\right),
$$

where $\psi_{j}^{+}$is the extended version of $\psi_{j}$, obtained by adding elements from $W^{+}$. For example, suppose $W^{+}$includes the physical objects in $W$ as well as other more abstract, mental objects. Then $\psi_{t}$ represents the state of agent $X$ 's body at time $t$, then $\psi_{t}^{+}$is that of his body-mind at that time. Here $\psi_{t+1}$ is implicitly determined by $L^{+}$acting on the enhanced state $\psi_{t}^{+}$. In this case, there is no $\mathrm{FW}$ in $W^{+}$, and in an absolute sense, no $\mathrm{FW}$ at all, since the deviations from $L$ are deterministically determined by the higher law $L^{+}$. Another possibility is that there is a probabilistic law $L^{+\prime}$ that governs the $W^{+}$objects, but here again there is no FW, as argued in Section III By the Weak Free Will paradox, which is adapted to $W^{+}$agency, there is FW only if there can be deviations from law $L^{+}$. 
The causes (or parameters) of these deviations are objects that live in a yet higher world $W^{++}$. It is clear that the above argument relativizes, i.e., one can recursively apply the above argument, shifting the level from $W^{+}$to $W^{++}$, and beyond. If this recursion is finite, then although there is FW in the sense of the Weak Free Will paradox till that causal depth, still there is none beyond. What remains just above that depth is either determinism or indeterminism. In other words, the Strong Free Will paradox is the argument that the only fundamental causal primitives are determinism and indeterminism. There is no room for any primitive like FW, which is seen ultimately to be a hierarchical interplay of these two primitives.

The quantum homeostatis hypothesis [17] and the FUN model [4], which provide a resolution to the Weak Free Will paradox, do not appear to get past the Strong Free Will paradox. In the former case, the Strong Free Will argument applies to the resource by which an agent controls the ordering of outcomes, and in the FUN model, it applies to the freedom paramter $\varphi$.

\section{FREE WILL AS INFINITE META-THEORETIC CONSTRUCT}

We propose that the response to the Strong Free Will paradox is to simply embrace it! We suggest that it is in the nature of Consciousness that it can support the above causal recursion to any depth. For convenience, let the base (physical) laws $L$ now be denoted by $L^{(0)}$ and the corresponding physical world $W$ in which they operate, by $W^{(0)}$. The usual laws of physics are order-0 laws, which govern the behavior of order-0 objects, which are physical objects, denoted $\lambda^{(0)}$, such as the physical component (body) of sentient agents like humans. The physical state of such a being is denoted $\psi^{(0)}$.

Volition- or will-driven deviations (if they exist) from the order-0 laws are caused, according to Eq. (1), by order-1 objects in $L^{+}$, which we denote now by $L^{(1)}$. These objects, denoted by $\lambda^{(1)}$, live in the larger world $W^{+}$, denoted by $W^{(1)}$. We have $W^{(0)} \subset W^{(1)}, \lambda^{(0)} \in W^{(0)}$ and $\lambda^{(1)} \in W^{(1)}-W^{(0)}$. The extended state of the free-willed being $X$ is $\psi^{(1)} \in W^{(1)}$ such that $\psi^{(0)}=\psi^{(1)} \cap W^{(0)}$. Similarly, will-driven deviations from $L^{(1)}$ dynamics are attributed to causes $\lambda^{++} \equiv \lambda^{(2)} \in W^{++} \equiv W^{(2)}$. The law $L^{(1)}$ is a meta-law for $L^{(0)}$ in that it is law that is about and governs $L^{(0)}$. The objects in $W^{(1)}$ and the axioms of $L^{(1)}$ thus constitute a meta-theory $\mathcal{T}^{(1)}$ for the base theory $\mathcal{T}^{(0)} \equiv\left(W^{(0)}, L^{(0)}\right)$.

At each level $j$ (a positive integer), the pair $\left(W^{(j)}, L^{(j)}\right)$ constitute the order- $j$ meta-theory $\mathcal{T}^{(j)}$. Formally, this system of meta-theories $\mathcal{T}^{(j)}$, can be continued indefinitely. The meta-theoretic construction of FW is the assertion that at each level $j, \mathrm{FW}$ at that level is expressed as the possibility of deviation from $L^{(j)}$ caused by order- $(j+1)$ objects $\lambda^{(j+1)} \in W^{(j+1)}$. Relative $\mathrm{FW}$ exists at level $K$ if there are deviations from all $L^{(j)}$ with $j<K$. If there are no deviations in $L^{(K)}$, then there is no FW beyond order- $K$. We propose the existence of absolute FW exists in the sense that relative FW exists for all finite $K$. In other words, no matter how deep we go tracking down causes, the causes of these causes, the causes of causes of causes, and so forth, although our explanatory power may increase, still there remain unexplained deviations.

We note that determinism and indeterminism lack this meta-theoretic character in that they can be described within a given level (say $L$ ) of the dynamics. Free will then is a new causal primitive, in addition to determinism and indeterminism. But although it is not logically necessary for indeterminism to be meta-theoretic, still it may be a function of higher-order causes. It differs from free will at the same order in not being correlated with the immediately higher order meta-theory. Thus indeterminism is just freedom without the will. Quantum fluctations are then order-0 freedom, while sentient beings who deviate from $\hat{N}$ in the FUN model possess (at least) order-0 free will.

If no such correlated parameter is evident in the meta-theory, then there is freedom alone, and to ensure that causality holds, we conclude that the causes lie in meta-theoretic depths farther down. This is a subtle but socially significant difference between the free will of sentient agents and the freedom of particles. Although there is no logical necessity to do so, we propose that all indeterminism is freedom in the above sense.

A problem that merits further study is the question of how the different meta-theories $\mathcal{T}^{(j)}$ may be demarcated. One may consider that $\mathcal{T}^{(0)}$ corresponds to predicate logic, and $\mathcal{T}^{(j)}(j>0)$ correspond to higher-order logics [20]. An example of a demarcation that doesn't work is the proposal that $\mathcal{T}^{(0)}$ is classical mechanics and $\mathcal{T}^{(1)}$ quantum mechanics, the idea being that quantum mechanics causes deviations from classical behavior. We venture that quantum mechanics, and thus also classical mechanics, belong in $\mathcal{T}^{(0)}$, the base physical theory. Intuitively, this is because quantum mechanics can be considered as a modification over an indeterministic generalization of classical mechanics. Although this modification is responsible for peculiarities like quantum nonlocality, it does not seem to endow quantum mechanics with more explanatory power, as we clarify below. 


\section{COMPUTABILITY AND CAUSALITY}

We suggest that the different levels of theory $\mathcal{T}^{(0)}$, the meta-theory $\mathcal{T}^{(1)}$, the meta-meta-theory $\mathcal{T}^{(2)}$, etc. are distinguished in different levels of computability that they support. Computability, which is the capacity to effectively solve a problem, is a topic studied in mathematical logic and computer science. Computability theory is concerned with the question of whether an algorithm or effective procedure exists to solve a problem. It is known that there exist algorithmically uncomputable problems, e.g., the halting problem for computer programs (formally: Turing machines) [21]. One can conceive qualitatively more powerful computers (formally: oracle machines) that can solve these problems, but must contend with their own halting problem. One can continue this exercise, to build a hierarchy of ever more powerful oracle-machines and ever harder uncomputable problems. We propose that the different $\mathcal{T}^{(j)}$ 's correspond to the different rungs in this hierarchy.

We can now make precise the sense in which FW is said to exist in this model. In computation theory, an oracle for problem $A$ is a hypothetical device that given any instance of $A$ returns the answer in finite time. If a Turing machine with access to this oracle can solve every instance of problem $B$, then $B$ is Turing-reducible to $A$. In other words, if $A$ is computable, then so is $B$. If similarly, $A$ is reducible to $B$, then $A$ and $B$ are equivalent. A Turing-degree is the set of all problems that are mutually Turing-equivalent.

The set of all computable problems is a Turing degree, which quantifies the level of its algorithmic unsolvability. A higher Turing degree is the set of problems Turing-equivalent to the halting problem, for example, the problem of whether a statement can be proved from the axioms of set theory. This latter set is not solvable (with Turing machines). One can construct higher Turing degrees by relativizing the proof of the halting theorem for Turing machines to Turing machines with oracles.

Thus the different meta-theories correspond to different Turing degrees, with a higher-order theory being of higher Turing degree. The move from $\mathcal{T}^{(j)}$ to $\mathcal{T}^{(j+1)}$ is equivalent to a Turing jump [22], i.e., produces a unit shift in the Turing degree.

By this criterion, since the quantum version of Turing machine is not more powerful than a classical machine from a computability perspective (and even computational complexity perspective), quantum mechanics belongs to the same-order theory as classical mechanics, namely $\mathcal{T}^{(0)}$. On the other hand, a deterministic hidden variable theory for quantum mechanics is a likely candidate for $\mathcal{T}^{(1)}$. For one, it can deterministically explain deviations from classical mechanics in terms of hidden variables. Further, the ability to manipulate this subquantum information leads to super-Turing power in a computational complexity sense (solving NP-complete problems in polynomial time) [23].

Our assertion of absolute FW above can now be re-stated thus: it exists in the sense that the problem of determining an agent's free choice is of infinite Turing degree, i.e., for every oracle machine at finitely high order, there will be uncomputable residual deviations in a model of free choice. In other words, FW is not just uncomputable, but infinitely so!

The first hint that the this linking between causality and computability is an appropriate direction to proceed is the observation that the notion of proof in Gödel's incompleteness theorem 24] and of truth in Tarski's undefinability theorem [25], are, in some ways, similar meta-theoretic concepts. Gödel incompleteness may be considered as an avatar of Turing uncomputability [21], as we show in Section VIII]

\section{TRUTH, PROOF AND FREEDOM}

Imagine a human agent $X$ equipped with the usual cognitive apparatuses of a physical brain and something else called mind. The physical aspect of $X$ lives in world $W^{(0)}$ governed by physical law $L^{(0)}$, while the higher aspects of $\psi^{(j)}$ are attributed to the mind entity. Now $\mathcal{T}^{(0)}$ naturally determines a model of computation, and correspondingly the limits of computational complexity and computability, in the world $W^{(0)}$. If $\mathcal{T}^{(0)}$ is the physical world, then the relevant model is Turing machines (TMs) or their equivalent. All TMs, including the TMs representing agents $X, Y, Z$ et al. that live in $W^{(0)}$, can be enumerated according to a fixed scheme, e.g., Gödel numbering or Turing numbering. We denote the corresponding numbers $\underline{X}, \underline{Y}, \underline{Z}$ and so on.

We now present a proof of Gödel's theorem via the uncomputability of a problem that is equivalent to the halting problem [21]. Suppose algorithm $\mathcal{A}$ exists that can predict in finite time agent $X$ 's binary choice upon input $i$ :

$$
\mathcal{A}(\underline{X} ; \underline{i})=\left\{\begin{array}{l}
0 \Longleftrightarrow X(i)=0 \\
1 \Longleftrightarrow X(i)=1
\end{array},\right.
$$

i.e., the machine outputs 0 (1) if $X$ outputs 0 (1) acting on input $i$. We construct the following program:

$$
\mathcal{R}(p)=\left\{\begin{array}{l}
1 \Longleftrightarrow \mathcal{A}(p ; p)=0 \\
0 \Longleftrightarrow \mathcal{A}(p ; p)=1
\end{array}\right.
$$


where $p$ is a positive integer. By construction, $\mathcal{R}$ exists if $\mathcal{A}$ does. Applying $\mathcal{A}$ to $\mathcal{R}$ we have from Eqs. (8) and (9):

$$
\mathcal{A}(\underline{\mathcal{R}} ; p)=\left\{\begin{aligned}
1 & \Longleftrightarrow \mathcal{A}(p ; p)=0 \\
0 & \Longleftrightarrow \mathcal{A}(p ; p)=1 .
\end{aligned}\right.
$$

We obtain a contradiction when we set $p=\underline{\mathcal{R}}$, which is an instance of the diagonal argument, first pioneered by Cantor. The conclusion is that a general, sound predictive algorithm $\mathcal{A}$ is logically impossible because it would help create an algorithm that is so powerful that, knowing its own future, it could act contradictory to its own prediction.

Therefore, if the system $F$ of reasoning embodied by TMs in consistent, then to avoid the contradiction, $\mathcal{A}(\underline{\mathcal{R}} ; \underline{\mathcal{R}})$ loops infinitely. Therefore, $\mathcal{R}(\underline{\mathcal{R}})$ is undecidable within $F$ - a Gödel sentence for the system. How do we, as human beings reading this, know this truth of an algorithmically undecidable statement like $\mathcal{R}(\underline{\mathcal{R}})$ ? Can the human mind therefore be more powerful than TMs? According to a much debated anti-mechanist view due to Lucas [26, 27] and Penrose [28, 29], it is.

An objection [30] is based on Gödel's second incompleteness theorem. The reasoning that led us to the claim that "If $F$ is consistent, then $\mathcal{R}(\underline{\mathcal{R}})$ is non-terminating" can itself be programmed into the action of a TM. Thus if $F$ is in fact consistent, then the proof of its own consistency must be uncomputable, in order that $\mathcal{R}(\underline{\mathcal{R}})$ be unprovable in $F$. In this objection to the anti-mechanist view of the mind, even if human agents were consistent, they would be unable to establish this, and thus attain to a meta-mathematical comprehension of what TMs can't. To this view, the following counter-objection may be given: Suppose a large number $n$ of persons toss a fair coin each, assuming that humans are consistent if a head turns up, and that we are inconsistent otherwise. Then it follows that if it so happens that human beings are consistent, the understanding that $\mathcal{R}(\underline{\mathcal{R}})$ is non-terminating would make the mind will be more powerful than TMs in the case of those people in the group for whom $(n / 2$ in number, with exponentially high probability) a head turns up! Clearly, we wouldn't want to power of the mind to depend the whim of a coin! (On the other hand, if humans happen to be inconsistent, then the above argument fails.) Thus it seems that objections based on consistency of $F$ do not satisfactorily undermine the anti-mechanist view.

Another criticisim [31] is that it may not be possible to humanly construct a Gödel sentence for something so complicated as the TM representing a human being. However this objection seems to assume that the human may in principle be more powerful than a TM, but not in practice. The claim for the disputing the anti-mechanist view should, it would seem, not depend on limitations imposed on human beings like mortality, emotionalism, fatigue, etc., unless it can be demonstrated that such a factor will necessarily (logically) intervene to prevent a human from computing the Gödel sentence in question.

Yet another objection to the anti-mechanist view is due to Whiteley [32], which is that humans would themselves be prone to a Gödel sentence, not unlike TMs. Consider the following predicate formula, formalized suitably:

$$
\mathrm{P}(\xi) \equiv \text { " } \xi \text { cannot consistently believe this statement." }
$$

Now $P(X)$ is true or false. If true, then $X$ does not believe $P(X)$ when presented with it, making him incomplete for disbelieving a true statement. If $P(X)$ is false, then $\neg P(X)$ is true; in other words, he believes a false statement, making him inconsistent. To avoid inconsistency, we select for him to be incomplete. This is just an informal version of Gödel's theorem, with disbelief [33] self-referenced, rather than unprovability (Actually, presenting $P(X)$ to $X$ creates a double self-reference: in $X$ and in the resulting statement 34]). According to this objection, humans have similar limitations as those attributed to machines on basis of Gödel's theorem.

$P(X)$ can be consistently believed by agent $Y$, and $P(Y)$ by $X$. Now imagine that $Y$ believes $P(X)$ and informs this statement to $X$. As predicted $X$ is unable to believe $P(X)$, being consistent. When $Y$ asks him if he believes $P(X), X$ replies in the negative. When $Y$ asks him why, $X$ says that he cannot say exactly why, but somehow $P(X)$ feels unconvincing. Now $Y$ happens to be a mathematician, and explains Gödel's theorem to $X$ enlightening $X$ as to why $X$ is unable to believe $P(X) . X$ is now able to reflect upon the nature of his understanding and the limitations imposed by the theorem. He trusts $Y$ enough to know that $Y$ wouldn't believe $P(X)$ unless it were true. He then has an 'Ah ha' moment, and decides that $P(X)$ is true after all. His brain still disbelieves $P(X)$, but somehow he knows that $P(X)$ is true, though maybe he can't help acting as if it were false. If $X$ could not have this Ah-ha moment, he could well be a machine, and in social terms, he must be some kind of zombie or dull-witted person, not a normal human! Thus, the power of intuitive grasp seems to be that $X$ can supply his own meta-theory on the fly, and it is this power to meta-jump that seems to come from nowhere but the nature of consciousness, and divides man and machine!

In other words, $X$ is able to meta-straddle: he can both believe and disbelieve $P(X)$ simultaneously, without being inconsistent because these states occur at two different layers: the disbelief at $W^{(j)}$ (possibly $j=0$ ) and the belief at $W^{(j+1)}$. (Here we may recollect moments where our eyes/brain are deluded by an optical illusion which we know is illusory; or when we know that something is false at a higher level, and yet can't help believing it!) This conclusion is consistent with our demarcation of the meta-theories $\mathcal{T}^{(j)}$ in terms of uncomputability occurring in the theory [34]. 
Now it is conceivable that the reasoning that led us to the conclusion that if $X$ intuits the Gödel sentence, and then meta-straddles, can itself be formalized or equivalently mechanized somehow, for example having a multi-tape TM with a denial on the lower tape, and acceptance on a higher tape that accesses an oracle. So this order-1 oracle machine will be our new model for agent $X$. However, one can construct a Whitely sentence $P^{(1)}(X)$ for $X$ modelled as an oracle-1 machine, and repeat the above argument, for $X$ perceiving the truth of this sentence. Relativizing this procedure, we construct ever more powerful oracle machines, going up the jump hierarchy, and iterating farther into transfinite ordinals, and even uncountable regular cardinals [22]. In each case, $X$ modeled as these machines still makes the intuitve jump, because if it didn't, we would call it a (higher-order) zombie. Whereas the very character of a machine must be changed as we climb up the jump hierarchy, the consciousness of $X$ seems essentially to be the same thing, as it jumps up or hops outside endlessly. It has the magical infinitely, meta-expansive plasticity that seems to be the essence of conscious understanding, as against mechanized assimilation by an automaton.

\section{MIND AND MAGIC: POSSIBLE RAMIFICATIONS FOR AI, PHYSICS AND NEUROSCIENCE}

Without going into details, we may assume that the relation between the physical aspect and the mind of human agents is causal both ways, i.e., that each influences the other. We may deduce that there are oracles located in the brain, that serve as gateways through which $\lambda^{(1)}$ and higher influences can flow into the physical system, to produce deviations from $L^{(0)}$ laws, giving rise to free will in the physical plane. Since our conscious actions are arguably not more powerful than TMs, it follows that the influence of these brain oracles must occur at a subconscious or unconscious level. Therefore, the only failsafe way to access this super-Turing computational capacity involved in a person's free choice is to present the person with the choice, and allow him to react spontaneously. To use a Biblical allusion, there was no way to compute what Adam's reaction would be to the injuction forbidding him to eat the apple in the forest of Eden, except by putting him physically in the forest and forbidding him! From a computational perspective, every instance of free choice is a miracle! These observations have various ramifications discussed below.

Our model leads naturally to a Cartesian mind-body dichotomy, where the mind is indefinitely layered into increasing levels of uncomputability. Since the conscious body/mind is no more powerful than TMs, the entirety of the human Consciousness cannot be consciously grasped through a finitary reasoning process, and must be done intuitively. From the perspective of the physical world, the actions of a free-willed agent are not always predictable, without being random. Mathematically, free choice can be described probabilistically as in Eq. (4) of the FUN model, where probability arises through unknowability. Any AI algorithm or neural network system, being no more powerful than Turing machines, can never in general simulate a free choice of human or presumably even an animal or, in general, any entity 'vivified by a soul'. Thus Consciousness, of which intuitive thought and FW are aspects, must also be also unsimulable, in contradistinction to the premise of Strong AI.

It seems possible that phenomena like savantism or the pattern recognition in the brain make use of the higher computational capacity attributed to the subconscious mind in our model. There must be something special in the structure of the brain that allows an interface with a subconscious oracle, a point that we discuss elsewhere [3]. We venture that the above phenomena are windows on that structure.

The free-willed human being is not an isolated entity, but part of the universe. If $\mathrm{FW}$ has the above implications for the physics of the human brain, this must have some implications, significant or otherwise, for the physics of the universe at large. If we extrapolate the above of hierarchy of laws to the large scale, then the grand unified theory (GUT), when discovered, will be simply be the tip of an indefinite hierarchy of meta-theories. We can then ask whether a notion of FW can, in a sense, be considered on a larger, cosmological scale. Perhaps spontanenous symmetry breaking and even the Big Bang, were possibly free choices by the 'mind of the universe'. Finally, given the greater computability supported the higher-order laws, it is impossible for physical laws to 'compute' them into existence. Thus the Big Bang may well be the 'ground zero' of a Bigger Bang followed by a Turing cascade!

\section{CONCLUSIONS AND DISCUSSIONS}

We highlighted the logical paradoxes encountered in defining FW and understanding its role in physics. We highlight our main conclusions. A principal conclusion is that like the concept of truth in Tarski's undefinability theorem, free will is also meta-theoretic: it correlates freedom to deviate from physical law in a base theory to parameters of 'will' in a meta-theory. Next, we pointed out that this meta-theoretic coupling must be continued infinitely in order to construct the FW that we intuitively associate with sentient agents. Third, the meta-theories are shown to correspond to a hierarchy of uncomputability. A consequence is that free will is uncomputable to infinite degree, in the sense that for a meta-theory at any finite order, there will deviations from its laws that are uncomputable in it. We justify this link between causality and computability by revisiting self-referential logical paradoxes that serve as the basis 
for theorems of Gödel-Tarski kind. Finally, we present a refinement of an anti-mechanist argument for consciousness based on these theorems.

[1] L. Zagzebski. Free will and foreknowledge, in Stanford Encyclopedia of Philosophy, edited by E. N. Zalta (2011), URL plato.stanford.edu/entries/free-will-foreknowledge/

[2] T. O'Connor. Free will, in Stanford Encyclopedia of Philosophy, edited by E. N. Zalta (2010), URL plato.stanford.edu/entries/freewill/

[3] H. Hanaan and R. Srikanth, On the neurological basis of free will: Locating the Gödel oracle (2013), under preparation.

[4] Chetan S. Mandayam Nayakar, S. Omkar, R. Srikanth. Consciousness, Libertarian Free Will and Quantum Randomness, in Interdisciplinary Perspectives on Consciousness and the Self (Eds. S. Menon, A. Sinha and B. V. Sreekantan) pp 307-323 (Springer 2014).

[5] V. Vedral, New Scientist 18 November, 55 (2006).

[6] A. Zeilinger (2006), interview in Die Weltwoche, Ausagabe 48/05.

[7] G. 't Hooft (2007), quant-ph/0701097.

[8] N. Gisin (2010), arXiv:1011.3440.

[9] H. Everett, Rev. Mod. Phys. 29, 454 (1957).

[10] J. Conway and S. Kochen, Found. Phys. 36, 1441 (2006), arXiv:quant-ph/0604079.

[11] J. Conway and S. Kochen, Amer. Math. Soc. 56, 2 (2008).

[12] N. Gisin, arXiv:1002.1392 (2010).

[13] R. Tumulka, Found. Phys. 37, 186197 (2007).

[14] S. Goldstein, D. V. Tausk, R. Tumulka, and N. Zanghi (2007), arXiv:0905.4641.

[15] A. Suarez (2010), arxiv:1002.2697.

[16] M. J. W. Hall, Phys. Rev. Lett. 105, 250404 (2010).

[17] A. Suarez (2008), arxiv:0804.0871.

[18] J. F. Clauser, M. A. Horne, A. Shimony, and R. A. Holt, Phys. Rev. Lett. 23, 880 (1969).

[19] A. Suarez (2010), arxiv:1006.2485.

[20] H. B. Enderton (Stanford University, 2008).

[21] M. Davis, Computability and Unsolvability (Dover, 1982).

[22] R. A. Shore and T. A. Slaman, Math. Res. Lett. 6, 711722 (1999).

[23] A. Valentini, Pramana - J. Phys. 59, 269 (2002).

[24] K. Gödel, Monatshefte für Mathematik und Physik 38 (1931).

[25] A. Tarski, Philosophy and Phenomenological Research 4 (1944).

[26] J. Megill, in Internet Enclopedia of Philosophy, edited by J. Fieser and B. Dowden (2012), URL www.iep.utm.edu/lp-argue.

[27] J. R. Lucas, The Freedom of the Will (Oxford University Press, 1970).

[28] R. Penrose, The Emperor's New Mind (Oxford University Press, 1989).

[29] R. Penrose, Shadows of the Mind (Oxford University Press, 1994).

[30] H. Putnam, in Dimensions of Mind. A Symposium, edited by S. Hook (London: Collier-Macmillan, 1960).

[31] P. Benacerraf, Monist 51, 9 (1967)).

[32] C. Whiteley, Philosophy 37, 61 (1962).

[33] D. M. Mackay, Mind 69, 31 (1960).

[34] R. Srikanth, Uncomputability, causality and cognition (2013), under preparation. 